\title{
The tale of two lexicons: Decomposing complexity across a distributed lexicon
}

\author{
Terje Lohndal ${ }^{1,3} \&$ Michael T. Putnam ${ }^{2,4}$ \\ ${ }^{1}$ NTNU - Norwegian University of Science and Technology \\ ${ }^{2}$ Pennsylvania State University \\ ${ }^{3}$ UiT The Arctic University of Norway \\ ${ }^{4}$ University of Greenwich
}

May 17, 2021

\begin{abstract}
The notion of complexity is evasive and often left to intuition, yet it is often invoked when studying heritage language grammars. In this paper, we propose a first pass at decomposing the notion of complexity into smaller components in a formal grammatical model. In particular, we argue that a distributed model of the lexicon (i.e., one that assumes that principles that generate words and phrases are one and the same) allows us to identify three components: syntactic features, the hierarchical organization of features, and the mapping between syntactic features and their exponents. Based on grammatical gender in different language pairs, in particular American Norwegian, we illustrate how this can account for developments in heritage language grammars whereby the grammatical gender system is considered to become less complex. More
\end{abstract}


generally, the paper demonstrates how a distributed architecture is better suited empirically and theoretically as a heuristic to understand complexity effects in heritage grammars and beyond.

\section{Introduction: What is complexity anyway?}

It is often argued that heritage languages exhibit reduced complexity, for instance through loss of morphological richness (Benmamoun, Montrul, \& Polinsky, 2013; Montrul, 2016, Polinsky, 2018) or by employing fewer syntactic rules (Polinsky, 2011; Westergaard \& Lohndal, 2019). The following two statements are representative of this general intuition that has often been expressed in the literature.

If complexity is something that can be measured consistently, then we might expect heritage languages to consistently exhibit reduced complexity and thus reduced expressive power compared to the native baseline. (Scontras, Fuchs, \& Polinsky, 2015, 17)

[...] maintaining two grammars in parallel creates an additional challenge [...] heritage speakers are likely to face difficulty with phenomena that impose relatively high cognitive demands...heritage speakers could face pressure to restructure their grammar in a way that frees up processing resources. (Polinsky \& Scontras, 2020, 14)

However, the available literature makes it clear that the picture is notably more complicated. First, it is not clear that the entire grammatical competence of heritage speakers should be labeled as 'complex' or 'less complex' relative to a given baseline. Rather, a certain pocket of the grammar may be less complex than the baseline. Second, even if one 
area is less complex, another may actually retain its complexity, as the following quote conveys.

[...] some heritage grammars actually maintain significant complexity in spite of being acquired under conditions of reduced qualitative and quantitative input. (Bousquette \& Putnam, 2020, 189)

Put differently, it is not a given that complexity in heritage languages is strictly relegated to wholesale simplification or reduction of attributes of a particular language. Third, if complexity in one area is reduced, such a reduction may lead to increased complexity in a different area. For instance, if the number of realized gender distinctions is reduced, the mapping between gender and declension class may become more complex, thereby also affecting the possible predictive relationship between the two.

This brief introduction suggests that 'complexity' is a variable notion, and in turn, that it may not be a primitive concept. In this paper, we will argue that in modeling what at face value appears as 'complexity' in any reliable sense, we need to decompose the notion of complexity in order to achieve a fuller understanding of its intricate nature in grammars. That is, we will illustrate how complexity can be deconstructed into parts of a formal grammatical system. We demonstrate the necessity and polyfunctionality that formal approaches to grammar, in particular those that advocate for the realization of morphology after the construction of syntactic structure, in capturing the effects of 'complexity' in heritage language grammars. As a first pass, we suggest that there are three concepts that are crucial in modeling complexity in a formal system: (i) syntactic features, (ii) the hierarchical representation of said features, and (iii) the mapping between said features and morphophonological exponents. We will show how such a view dovetails naturally with a particular distributed model of the lexicon. This heuristic will be applied in modeling the phenomenon of grammatical gender in heritage grammars. 
The paper is structured as follows: In $\$ 2$ we outline the core desiderata and assumptions that accompany a distributed view of the lexicon, demonstrating how this is an especially ideal fit for bi/multilinguals. We establish a working definition and heuristic for decomposing complexity in $\$ 3$, showing how this is able to capture insights in the distribution of mixed determiner phrases (DPs) found in American Norwegian (AmNo) in $\$ 4$ $\$ 5$ summarizes and concludes the paper.

\section{Heritage grammars and a distributed lexicon}

The field of linguistics, like any other field of scientific inquiry, is often cluttered with terminology that is frequently used by many, yet poorly understood by the majority of people that use said terms. A common contributor to these terminological misconceptions stems from ongoing debates and controversies surrounding the best way to categorize and summarize key concepts. In this paper, we examine one of these controversial concepts, the lexicon, and its relationship with yet another classic thorn in information theory, the notion of complexity. Arriving at a functional definition of what a lexicon is (and isn't) is paramount for any generative (here meaning formal and explicit) approach to linguistic structure, because it provides a clear demarcation of which elements of structure are stored and which are generated.

The controversy surrounding the difficulty in establishing a clear-cut definition of the term lexicon often boils down to one's view of the role of morphology in a particular framework. To put it bluntly, what does lexical knowledge consist of? Embick (2015, 14) lists three different notions of lexical:

\section{(1) Notions of lexical}

a. The idea that the lexicon is a generative system in which words (as opposed to 
syntactic objects) are derived.

b. The idea that basic elements (morphemes) must be listed, because they are underived.

c. The idea that the unpredictable behavior of complex objects must be listed.

Although most models of morphology adopt some version of (1p) and (1k), there exists a significant divide between models that advocate for the existence of a separate, often 'pre-syntactic' lexicon where words are generated prior to their inclusion in syntactic operations, and those that adopt alternative approaches. Models that call for a pre-syntactic lexicon contrast with those often called 'single engine' approaches, whereby morphological and syntactic structures are generated (largely) by the same principles. Models that endorse the view of a separate, pre-syntactic lexicon are referred to as lexicalist, or endoskeletal frameworks (Bresnan \& Mchombo, 1995; Ježek, 2016, Müller \& Wechsler, 2014; Spencer, 2005; Wechsler, 2015), while in contrast, those that adopt the 'single engine' approach are known as non-lexicalist, or exoskeletal models (Borer, 2005a, 2005b, 2013; Bruening, 2014, 2018; Embick \& Marantz, 2008; Embick \& Noyer, 2007; Halle \& Marantz, 1993).1. In these latter approaches, the linguistic information attributed to the lexicon is distributed throughout other modules of the architecture of grammar; i.e., words have no privileged status as atomic units in such a system. In this paper, we present a particular non-lexicalist implementation of the lexicon. However, first, it is necessary to outline some basic assumptions regarding the nature of the bi-/multilingual lexicon, which we turn to below.

\footnotetext{
${ }^{1}$ Note that the term 'exoskeletal' is often connected specifically to Hagit Borer's particular model. Here we use it as a more general term, in line with Grimstad, Riksem, Lohndal, and Åfarli (2018).
} 


\subsection{The integrated nature of bi-/multilingual grammar}

Much recent experimental work on the bilingual lexicon and grammar suggests that its structure is highly integrated (Green \& Abutalebi, 2013; Harstuiker, Pickering, \& Veltkamp, 2004; Starreveld, De Groot, Rossmark, \& Van Hell, 2014; Sudarshan \& Baum, 2019). Irrespective of the proficiency that bilinguals possess in each (competing) source grammar, an abundance of evidence confirms the highly integrated nature and continued competition of elements of grammar, including the lexicon; see Kroll and Gollan (2014) and Putnam, Carlson, and Reitter (2018) for a review of extant literature. Put differently, bi/multilinguals can best be described as multi-competent (Cook, 2016), possessing simultaneous access to elements of grammar from both/all source grammars. From a distributed lexicon perspective, the question becomes how such an integrated lexicon should be modelled. For example, are items from each language stored in separate lists, or is there just one list of items irrespective of which language they typically occur in (see Alexiadou and Lohndal (2018); López (2020) for discussion).

It is important to emphasize the impact an integrated bi/multilingual grammar system has on mental representations more generally. Experimental research over the past decade has demonstrated that comprehenders are able to make rapid adaptations at the sentencelevel (Branigan \& Pickering, 2017; Fine, Jaeger, Farmer, \& Qian, 2013), whose predictions of what structures may (or may not) come next are aided by the hierarchical structure of elements as they appear in syntax (Brennan \& Hale, 2019; Ding, Melloni, Zhang, Tian, \& Poeppel, 2016). It should therefore come as little surprise that experimental evidence also confirms that this hierarchical structure found in syntax also facilitates morphological processing (Gwilliams, 2019; Marantz, 2013; Oseki \& Marantz, 2020). Recent work by Stefanich, Cabrelli, Hilderman, and Archibald (2019) illustrates how a hierarchical approach to morphology provides valuable insight into the morphophonological and prosodic 
boundaries of code-switched structures, and Song, Do, Lee, Thompson, and Waegemaekers (2019) and Song, Do, Thompson, Waegemaekers, and Lee (2020) demonstrate the usefulness of utilizing hierarchical structure in measuring the differences in L2 morphological representations when compared with monolingual controls. Taken together, both sentences and words have internal hierarchical structure, and some distributional models, such as Distributed Morphology, hold that the principles governing both are the same.

\subsection{Arguments for a distributed lexicon}

A grammar with a distributed lexicon is one that divorces structure-building mechanisms from properties of lexical items. This interpretation of the lexicon contrasts with models that are inherently endoskeletal, i.e., those that have a pre-syntactic lexicon that houses separate generative operations prior to the insertion of 'words' into the syntax. Perhaps to the surprise of some, Chomsky's $(1955 / 1975$; 1957) earliest generative proposals were actually exoskeletal in design; Phrase Structure (PS) rules generated structures and lexical items were inserted once said structures were built, see (2).

(2) Sample Phrase Structure (PS) rules
a. $\mathrm{S} \rightarrow \mathrm{NP}$ VP
b. $\mathrm{NP} \rightarrow \mathrm{N}$
c. $\mathrm{VP} \rightarrow \mathrm{V}$
d. $\mathrm{N} \rightarrow$ John
e. $\mathrm{V} \rightarrow$ left

Setting aside some details, assuming a grammar with PS-rules such as (2) would result in a tree structure like (3) below: 
(3)

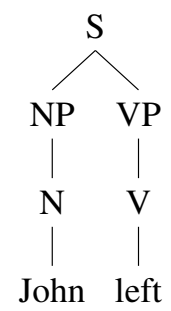

Therefore, it is fair to say that the basic guiding intuition behind generative frameworks that adopt some version of an exoskeletal architecture finds its origin its earliest versions. More recent instantiations of distributed architectures build upon this tradition, while advancing more exact and nuanced proposals about the nature of linguistic competence. These more recent approaches place an emphasis on the way in which syntactic structure determines both the grammar properties and "the ultimate fine-grained meanings of lexical items themselves" (Borer, 2003, 33). Again, this boils down to the core architectural design of exoskeletal grammars that support that view that syntax - and its core structure-building principles - is responsible for constructing the lexicon, and not the opposite scenario, where elements from the lexicon feed syntactic operations.

All exoskeletal approaches adopt some version of a realizational approach to morphology, where morphosyntactic properties license (i.e., determine) inflection morphemes (see Stump (2001) for an overview). Under this view, the syntax is composed of atomic units - roots and formal features that mark syntactic and semantic properties (i.e., synsemfeatures) - that map to morphophonological realizations, which we will call exponents in what follows. Exactly how this mapping occurs is a matter of controversy, with proposals ranging from Distributed Morphology (Halle \& Marantz, 1993) to Nanosyntax (Starke, 2009). We will not engage in that debate here, rather we will assume an architecture broadly consistent with Distributed Morphology here for implementational purposes.

Within Distributed Morphology, a Vocabulary Item denotes the mapping between ab- 
stract syntactic-semantic (synsem) features that are realized by phonological exponents. This is illustrated in (4) (adopted from Embick (2015, 9)):

\section{(4) Vocabulary Item:}

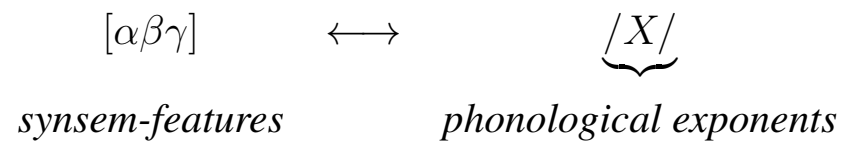

This dynamic and distributed view of Vocabulary Items (and the lexicon more generally) finds support in a number of empirical domains. For example, a substantial body of evidence has been mustered from the area of argument structure, in particular involving argument structure flexibility. Consider the the different environments that the root $\sqrt{\text { whistle }}$ can appear in (5):

(5) a. Kim whistled.

b. Kim whistled at the dog.

c. Kim whistled a tune.

d. Kim whistled a warning.

e. Kim whistled me a warning.

f. Kim whistled her appreciation.

g. Kim whistled to the dog to come.

h. The bullet whistled through the air.

i. The air whistled with bullets. (Rappaport Hovav \& Levin, 1998, 98)

In the examples above, $\sqrt{\text { whistle }}$ can appear as an unergative predicate $(5 \mathrm{a})$, a predicate that licenses a directive PP $(5 \mathrm{~b})$, a transitive predicate $(5 \mathrm{~b}, \mathrm{~d})$ with a cognate object, a ditransitive with a benefactive argument either as a DP (5) or as a PP (5 5 , and a transitive predicate $(5 \mathrm{f})$. In addition, inanimate subjects are also possible, as in $(5 \mathrm{~h}, \mathrm{i})$. Clearly, 
any descriptively adequate treatment of the argument structure of $\sqrt{\text { whistle }}$ must be fairly flexible to account for the well-formedness of the examples in (5). The primary question is, of course, what should we make of this flexibility? There are two primary options to consider: (1) We can assume an endoskeletal approach with a pre-syntactic lexicon that consists of eight different versions (i.e., lexical items), which is the approach that Rappaport Hovav and Levin (1998) take. This would require a way of properly discerning which of the eight lexical items should be used, resulting in an exuberant degree of polysemy in the lexicon, or (2) we adopt an exoskeletal approach which maintains that (verbal) roots do not have inherent argument structure that is projected into the syntax. The second option avoids unwanted polysemous lexical items being stored and then reprojected into the syntax. In turn, this approach to argument structure requires a handle on various restrictions *Lisa kicked, *Kathy gave Bill), and various proposals exist (Borer, 2005b; Lohndal, 2014).

This particular distributed bilingual lexicon model has already been applied to bi- and multilingual speakers from a diverse array of languages and populations Aboh, 2015; Alexiadou, 2017; Alexiadou \& Lohndal, 2018; López, 2020; Putnam, 2020; Putnam, PerezCortes, \& Sánchez, 2019; Riksem, 2017; Riksem, Grimstad, Lohndal, \& Åfarli, 2019). In the present paper, we take things one step further and demonstrate how adopting a distributed model of morphological knowledge enables us to identify some of the basic ingredients of the illusive notion complexity and its decomposition.

\section{Modeling complexity in heritage grammars}

Equipped with the understanding of how a non-lexicalist approach to grammar relies on a distributed lexicon, we put forward an initial proposal regarding how one can conceive of complexity in such a system, and particularly in heritage language grammars. We first 
turn to a brief review of some general and typological work on complexity in $\$ 3.1$, which then transitions into how complexity is not a primitive; rather, it should be decomposed into smaller components in $\$ 3.2$.

\subsection{Approaches to complexity}

Initial attempts to formally define complexity in science are certainly not unique to formal linguistics and can be traced back to initial proposals by Shannon (1948), and more recently there has been a renewed interest in understanding complexity from a linguistic, in particular typological, standpoint (e.g., Audring, 2014, 2017, 2019; Dahl, 2004; DeGraff, 2001; Di Garbo \& Miestamo, 2019; McWhorter, 2001; Miestamo, 2006, 2008; Newmeyer \& Preston, 2014; Sampson, Gil, \& Trudgill, 2009). The bulk of research on complexity from a typological perspective has focused on providing descriptive generalizations; however, the majority of them fall short of engaging in attempts to propose predictive outcomes. If, as recently stated by Polinsky and Scontras (2020), one of the next important steps in advancing research on heritage language grammars requires the development of a working notion of predicting grammatical outcomes (Polinsky \& Scontras, 2020, Putnam, 2020), we maintain that coming to terms with a heuristic of complexity is essential to this larger goal.

Our conceptualization of complexity as realized through a distributed lexicon developed here finds its roots in work by Miestamo (2006, 2008), whose fundamental axiom can be reduced to the number of 'elements' in a system, and the number of connections that exist between them. The challenge, of course, lies in properly defining the ontological status of these 'elements' within a particular system; however, this task is not too daunting in a distributed lexicon. As a point of departure, we can assume that the atomic synsem-features in Vocabulary Items as declared above in (4) function as these elements 
and their 'mappings' involved the proper identification with (morphophonological) exponents. An attractive feature of this proposal is that, from an information-theoretic point of view, complexity can be reduced to description length; i.e., the simpler entity can be compressed into a smaller space without facing the risk of losing information. An outcome of this proposal is that some synsem-feature-to-exponent mappings are held to be more complex than others, which has the advantage of delivering a falsifiable claim that can be rigorously examined in both on- and offline studies.

\subsection{A decompositional approach to complexity}

Not only does a bi/multilingual lexicon along the lines of our distributed description reflect a more accurate depiction of such a system, but it also establishes a more precise heuristic of tackling complexity and how this can be integrated into discussions of heritage language competence. The actual structure-building mechanisms and operations are assumed to be identical across all languages, involving the building of structure by two core operations: (i) Merge, which allows syntactic structure to be constructed hierarchically, (ii) The manipulation of synsem-features in local configurations by Agree (see Adger (2003) for a textbook exposition). Crucially however, what sorts of structures these functions operate over may differ considerably. This state of affairs yields two predictions: (i) the fewer functional (i.e., synsem) features that are active in a particular system yields a less complex grammar in toto, and (ii) one form-one meaning mappings are regarded to be simpler than alternatives.

From this perspective, complexity can be conceived of in terms of the shear number of features and their associated functional projections ${ }^{2}$, but equally importantly, core differences between languages reside in the mapping from synsem feature(s) to exponent(s) (as

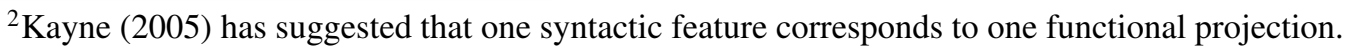


illustrated in (4)). These mappings are often not simplex and transparent (i.e., 1:1), and non-transparent mappings can be considered more complex (they are e.g., more difficult to acquire and master, due to the fact that they require more complicated rules). This falls out naturally from an exoskeletal architecture which allows us to separate the functional (synsem) features from their associated exponents.

In spite of the limited number of operations we propose, there are still certain challenges we face when attempting to operationalize complexity in such a model, that is, to identify the parts that together may be used to characterize complexity. This is a particularly acute matter when dealing with heritage language grammars. A primary challenge concerns determining which domains of grammar are considered to be more vulnerable to change and attrition (see, e.g., Montrul (2008, 2016); Polinsky (2018); Sorace (2011)). Domains of grammar that are vulnerable are not necessarily complex, and the inverse holds as well, i.e., elements of grammar considered to be more sturdy and less susceptible are not necessarily simple. For example, consider word order. As Polinsky (2018, 273) points out, "[...] word order appears to be a more vulnerable domain, subject to general change and sometimes transfer [...]". However, word order is often very salient in the input and not necessarily complex in terms of the number of rules required to derive its properties. On the other hand, resilient properties do not have to be simple. Verb Second (V2) is an example of this, which often involve different rules in main clauses and embedded clauses, and sometimes even for different types of $w h$-constituents (see Lohndal, Westergaard, and Vangsnes (2020) for discussion of Norwegian data which illustrate this point). Despite the complexity of the rules involved, $\mathrm{V} 2$ is often remarkably stable.

For these reasons, we acknowledge that any notion of complexity is best understood as a relative notion. Two examples come to mind to lend support to this claim. First, L2 speakers of English struggle to acquire subject-verb agreement, which is generally salient and transparently encoded. As White (2003, 187-193) points out, the accuracy of subject- 
verb agreement is between $4.5 \%$ and $46.5 \%$, whereas the accuracy is much higher for verb movement and subject expression. Second, in heritage language grammars, what is considered to be complex will also depend on the interacting languages of the dyad, and particular domains in which these two languages overlap and contrast with one another (Scontras \& Putnam, 2020).

These caveats notwithstanding, decomposing and operationalizing complexity through the lens of a distributed lexicon opens the door to a straightforward and conceptually appealing way to model this elusive, yet essential concept. As a point of departure, let us consider possible outcomes in heritage grammars. Our hypothesis is that these patterns help us identify core components of what we intuitively think of as complexity. Essentially, there are four possible outcomes. They are illustrated in (6).

(6) Relative to a given baseline, a feature can be
a. retained in the same hierarchical position
b. shift its hierarchical position
c. lost
d. (internally) restructured
i. loss of [some] features
ii. reconfiguration of features

Note that a lexicalist approach to the lexicon can also accommodate a change in the features of a lexical item, as well as adopting a rich functional sequence. Thus these two components are not unique to a distributed model of the lexicon. However, there are some core differences, which emerge more clearly in (6). The main difference is that the position of a feature is independent of the lexical item itself. This also means that a feature can shift its position in the functional sequence. Lexicalist approaches would in principle al- 
low for a change in the functional sequence, although not within words themselves, which is one of the advantages of a distributed architecture.

A distributed lexicon requires a distinction between the underlying features in a system, their subsequent values, and the actual exponents that are associated and matched with them. Such a distinction has been proposed both for second language learners and for language mixing in various kinds of bilingual speakers (Alexiadou, 2017; Alexiadou \& Lohndal, 2018; Lardiere, 1998, 2008; Lohndal, Rothman, Kupisch, \& Westergaard, 2019; Prévost \& White, 2000; Putnam, 2020; Putnam et al., 2019; Riksem, 2017, 2018; Riksem et al., 2019). In other words, no additional mechanisms or axioms beyond what we have proposed for the establishment of Vocabulary Items in (4) are necessary. In the next section, we will consider grammatical gender as a case study supporting the typology in 6 above.

\section{Application: Grammatical gender}

Here we shift gears to provide empirical support for our distributed model of a bilingual, heritage lexicon, and its advantages in establishing a model that can be used to operationalize systemic complexity. We will review data on grammatical gender from two heritage language situations: Spanish heritage speakers in the US and Norwegian heritage speakers in the US. In the latter case, we will also include data from mixed structures; i.e., units that possess elements from both English and Norwegian.

\subsection{Grammatical gender in heritage language grammars}

Given the complexity of gender (Corbett, 1991), the prediction is that heritage speakers face difficulties with grammatical gender. This prediction generally seems to be supported 
by the available data. Polinsky (2008) shows that more proficient speakers of heritage Russian in the US have retained a three-gender system whereas less proficient speakers only have a two-gender system. For the less proficient speakers, Polinsky (1997, 2006) already showed that these individuals do not fully master the complex system of declension classes. Instead, they rely on formal cues when assigning grammatical gender: Whether the noun in its base form ends in a consonant or a vowel. For AmNo, Lohndal and Westergaard (2016) show that the three-gender system of Norwegian is changing (see also Johannessen and Larsson (2015) and Rødvand (2017)). According to their observations, there is a general overgeneralization of the masculine, i.e., general erosion of the grammatical gender marking in system (leading to eventual loss). In this section we provide a detailed sketch of how gender is licensed in a distributed lexicon architecture, arguing that this provides important insights into how complexity could be decomposed into more basic units.

\subsection{The syntax of grammatical gender}

A first item of business is to address the where and how of grammatical gender in a distributed lexicon model. Since Picallo (1991) and Ritter (1993), an important research question has been to determine the structural locus of grammatical gender in syntactic structure. Based on her research on the syntax of gender, we adopt Kramer's (2016) review of these issues, supporting the view of gender being part of the noun stem. Specifically, gender features are located on the categorizer that provides category to a root, in the case of nouns the categorizing head $n$, represented in (7).

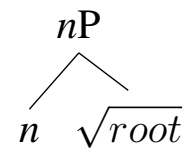


On this view, grammatical gender assignment involves an interplay between the genderassigning head $n$ and the particular roots that each such head can occur with. Below we will return to the issue of how gender assignment works within this framework. The essence is that grammatical gender assignment is syntactically represented, i.e., it is not simply a lexical property of an individual root or a stem.

\subsection{Restructuring the functional sequence}

On the view that grammatical gender assignment is a syntactic (rather than a lexical) property, the next logical question concerns how this feature is encoded in the syntax. In $\$ 4.2$ we argued that gender is located on the categorizing head $n$. An important and relevant question moving forward is whether gender, as well as other nominal features, such as number, are associated with the same functional heads in heritage speaker grammars and their relevant baseline(s). Investigating the morphosyntax of gender in heritage Spanish, Scontras, Polinsky, and Fuchs (2018) advance the claim that the functional sequence of elements in the DP differs between native and heritage speaker grammars. Adopting an agreement attraction paradigm, Scontras et al. (2018) propose that whereas native speakers maintain two separate heads for number and gender, these two projections are reduced to one in heritage grammars. They frame this outcome as being an instance of 'representa-

tional economy'. Concretely, native speakers employ separate [NUM] and [GEN] features and heads, which is illustrated in (8) \& (9) (see Fuchs, Polinsky, and Scontras (2015) for details):

(8) $\left[\mathrm{DP} \mathrm{D}\left[\mathrm{NumP} \mathrm{Num}_{[\mathrm{NUM}]}\left[\mathrm{GenP} \mathrm{Gen}_{[\mathrm{GEN}]}[n \mathrm{P} n]\right]\right]\right]$

In heritage grammars, the situation is different. The behavior of the speakers suggests that they have collapsed both the [NUM] and [GEN] features onto the same head, which is argued to be NumP. This is illustrated in (9). 


$$
\left[\mathrm{DP} \mathrm{D}\left[\mathrm{NumP} \mathrm{Num}_{[\mathrm{GEN}, \mathrm{NUM}]}\left[{ }_{n \mathrm{P}} n\right]\right]\right]
$$

The driving force behind this restructuring is likely due to "[...] feature opacity" leading "to interpretive instability", whose end-point may be that "the feature bundle might lose feature specification altogether" (Scontras et al., 2018, 21). This situation is not an isolated case in the study of heritage speaker grammar as confirmed in the pioneering research by Polinsky (1997, 2006, 2008), who demonstrates that features themselves may undergo restructuring. In terms of changes in complexity, Heritage Spanish is gaining complexity because it acquires a less transparent mapping from features to exponents. However, it is also becoming less complex in the sense that a smaller functional structure is generated. Our decompositional approach has the advantage that it naturally captures such opposing tendencies, yet an outstanding question is how this decompositional approach could be utilized to measure complexity. In this paper, we have to leave this question for future research.

In the following section, we take a closer look at another case of feature-restructuring, drawing on evidence from American Norwegian. These results will also illustrate that the mapping between gender and declension class may become less transparent as a consequence of restructured gender features, suggesting that the mapping between features and exponents are an important part of decomposing complexity.

\subsection{Restructuring features}

In this section, we take a closer look at the moribund heritage language American Norwegian, spoken today by only a few remaining elderly individuals. Even though it is a now moribund language, it has a rich history, which also is unusually well documented (Flom, 1903; Haugen, 1953; Hjelde, 1996; Riksem, 2018). The data are mostly based on previous work, but the specific analysis proposed is original to this paper. 
Before we turn to American Norwegian, we briefly outline some of the analytical assumptions we make regarding grammatical gender in Norwegian. Traditionally, Norwegian has a three-gender system consisting of masculine, feminine, and neuter distinctions. Table 1 outlines the various morphological reflexives of grammatical gender in a threegender variety of Norwegian.

\begin{tabular}{c||ccc}
\hline \multirow{2}{*}{ Indefinite } & Masculine & Feminine & Neuter \\
\hline \hline \multirow{2}{*}{ Definite } & en hest & ei seng & et hus \\
& h horse & a bed & a house \\
hesten & senga & huset \\
horse.DEF & bed.DEF & house.DEF \\
Double definite & den hesten & den senga & det huset \\
& DEF horse.DEF & DEF bed.DEF & DEF house.DEF \\
Adjectives & en fin hest & ei fin seng & et fint hus \\
& a nice horse & a nice bed & a nice house \\
Possessives & min hest & mi seng & mitt hus \\
& my horse & my bed & my house \\
\hline
\end{tabular}

Table 1: (Traditional) three-gender system in Norwegian (M/F/N)

Table 1 demonstrates that gender appears on multiple elements within the noun phrase, which is typically referred to as concord agreement. Formally this is generally implemented as agreement between the relevant functional heads, to which we return below.

With respect to the syntax of DPs in Norwegian, we adopt the hierarchy of projections depicted in (10) (see e.g., Julien (2005) for a similar structure). 


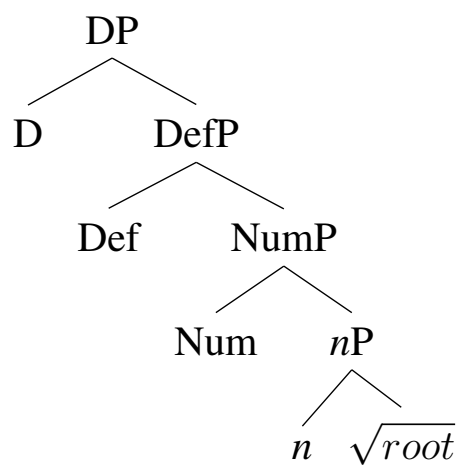

Working our way from the bottom up, at the base of this tree structure an uncategorized root merges with the categorizer $n$. The resulting projection, $n \mathrm{P}$, then merges with a head that encodes Number, yielding NumP. On top of NumP, the postnominal definite suffix is merged, as the head of DefP. And then the prenominal free-standing determiner is merged in D. Note that there may be additional projections, in particular between DP and DefP, which can host adjectives, numerals and possessives.

In what follows, we will focus on the $n$ head and its features, and following the analysis suggested by Lohndal and Westergaard (2021, 112) for Norwegian, which based on Kramer's (2015) approach, assigns features to $n$ heads. These features and their associated values are shown in (11).
a. $n[]$
(neuter)
b. $n[$ GEN: masc $]$
(masculine)
c. $n[\mathrm{GEN}: \mathrm{fem}]$
(feminine)

The assumption is that [GEN] is a multivalent feature whose values in Norwegian are either masculine or feminine (see Corbett (2012) for more on feature systems). Neuter does not have a feature specification as it is identified as the default gender feature.

On this approach, a categorizing $n$ head combines with a root to yield a particular noun. For instance, when $(11 \mathrm{~b})$ combines with the root bil 'car', the result becomes en bil 
'a.MASC car'. A core question concerns what governs the licit combinations of $n$ heads with roots. To address this issue from a Distributed Morphology perspective, Kramer (2015, 50-57) appeals to two types of licensing conditions: Semantic licensing conditions and arbitrary licensing conditions. The former are conditions that are related to the semantics of the root (and encoded in the Encyclopedia), whereas the latter are conditions unrelated to any property of the root. That is, they are idiosyncratic, "essentially a chance association between a root and a $n$ " (Kramer, 2015, 54).

There is a transparent relationship between gender features and the exponents for indefinite articles in Norwegian, as illustrated in(12).
a. $n[] \longleftrightarrow /$ e:t/
(neuter)
b. $n$ [GEN: masc] $\longleftrightarrow / \mathrm{e}: \mathrm{n} /$
(masculine)
c. $n[$ GEN: fem] $\longleftrightarrow /$ e:i $/$
(feminine)

Note, though, that these exponents also express indefiniteness. More generally, gender features exist in combinations (of features and their representative values) in the DP, i.e. the fact that there is concord between an indefinite article, an adjective, and the noun. As we will see in the next sub-section, this means that the system contains a lot of parts that need to be mastered. Combined with the fact that masculine also is by far the dominant gender in (American) Norwegian (Lohndal \& Westergaard, 2016, 2021), a prediction is that this kind of gender system may be more difficult to acquire and maintain in bilingual settings, in particular in heritage language settings.

As mentioned in $\$ 4.1$, the gender system in American Norwegian is changing. Here we will consider gender assignment on English nouns in mixed AmNo DPs specifically, which has been the object of study since the turn of the 20th century (see Flom (1903)). Generations later, Haugen (1953, 44) noted the generalization that "all nouns become masculine unless they were associated with a homophonous fem[inine] or neut[er] mor- 
pheme or a female creature.” More recently, Hjelde (1996) demonstrates that it is possible to identify morphological, semantic, and phonological rules for English nouns and also notes a rising trend in the assignment of masculine gender in these mixed DPs. Most recently, Riksem (2018) provides a detailed overview of the gender assignment on English words in AmNo. Her study, based on recorded speech in the Corpus of American Nordic Speech (CANS; Johannessen (2015)), examines 1265 nouns, with 1034 of them occurring Norwegian structures. Their distribution is reported in Table 2 .

\begin{tabular}{c|c}
\hline Masculine & $66.1 \%$ \\
Feminine & $6.5 \%$ \\
Neuter & $6.2 \%$ \\
Alternating & $21.2 \%$ \\
\hline
\end{tabular}

Table 2: Distribution of CANS data from Riksem (2018)

Examples (13) and (14) are examplars from Riksem (2018) of mixed indefinite and definite DPs respectively.

(13) Indefinite DPs
a. $\quad$ ei nurse (F) (coon_valley_WI_02gm)
b. et shed (N) (coon_valley_WI_02gm)
c. en chainsaw (M) (blair_WI_07gm)

(14) Definite DPs
a. field-a (F) (coon_valley_WI_02gm)
b. shed-et (N) (westby_WI_06gm)
c. chopper-en (M) (blair_WI_01gm) 
Many English nouns in AmNo no longer have the same gender as similar and identical nouns in Norwegian, although roughly $61.2 \%$ of the nouns have a gender that corresponds to the Norwegian equivalent. Divergent gender assignment was not ubiquitously manifest as masculine, with only $62.8 \%$ of these analyzed forms showing masculine. All others displayed different patterns of gender assignment. In many cases, it is difficult to discern exactly what the translations equivalents into Norwegian would be, which suggests that this cannot fully account for the assignment of grammatical gender (cf. Riksem (2018)).

Summarizing, across generations we see increased variability for all three genders and an increase in alternating gender. This shows that the grammatical gender system is vulnerable, most likely due to the lack of unambiguous cues for the three genders (cf. Lohndal and Westergaard (2016, 2021)).

Bearing these data in mind, we assume that AmNo speakers have a fixed indefinite article for the Norwegian noun (and that the suffixed article is generally target-consistent; cf. Lohndal and Westergaard (2016) and Johannessen and Larsson (2015)). English nouns in mixed DPs can be assigned grammatical gender (through Norwegian indefinite articles), and that all three genders found in Norwegian are represented to some degree. Considering the data, masculine is the dominant and default gender for English nouns. The assignment of feminine and neuter gender seems to be fairly random, depending on properties such as perceived phonetic similarity ${ }^{3}$ and semantic associations. Some speakers also appear to assign different genders to the very same root. Some of this variation is presumably found in the input of earlier generations of the speech of AmNo, due likely to the dialect variation that contributes to the variety. Another factor may be metalinguistic awareness, as most speakers of American Norwegian are aware that Norwegian typically has three genders,

$\sqrt[3]{\text { Haugen }}(1953,449)$ reports similar findings, stating "...in adapting the loanword to its new context, he might be easily be reminded of some native word of similar phonetic form which he could follow without qualms." 
which causes some of them to suffer from what has been described as 'language anxiety' (Eide \& Hjelde, 2015; Rødvand, 2017). That is, they are worried about not speaking Norwegian 'correctly' or 'properly' enough, which in this case amounts to not using all three genders correctly.

Let us now consider how an exoskeletal analysis can account for the variable assignment of gender to English words, building on work by Grimstad et al. (2018); Riksem (2018); Riksem et al. (2019). The tree structure below in (15) illustrates the structure a mixed AmNo DP consisting of a feminine indefinite determiner $e i$ 'the' and the English noun field prior to lexical insertion.

(15) DP in AmNo: ei field 'a field' (before lexical insertion)

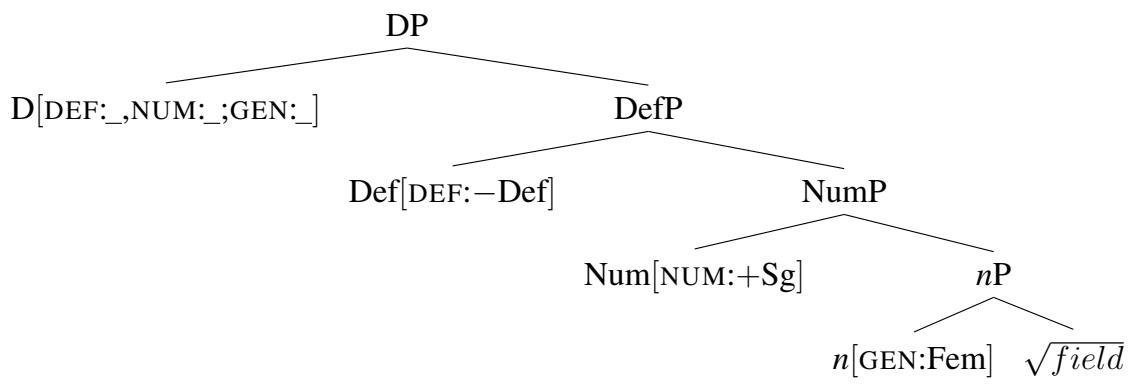

Although the values of the synsem-features for the functional heads $n$, Num, and Def are expressed, the values of these features remain undetermined on D at this stage of the derivation. Crucially, their value is determined by the syntactic configuration that they occur in, as illustrated in $(16)$ below. 
DP in AmNo: ei field 'a field' (after lexical insertion)

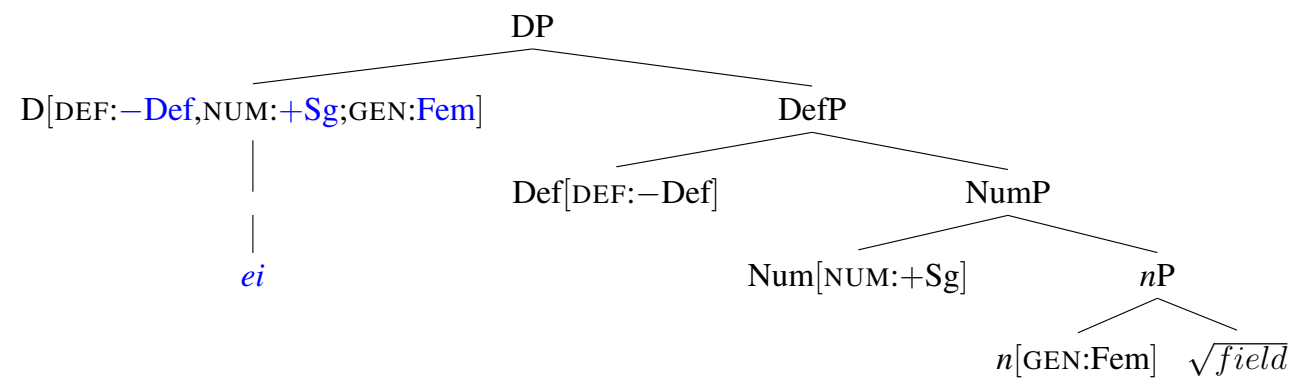

The licensing of gender can be captured by extending Kramer's $(2015,56)$ appeal to two kinds of licensing conditions. The latter can be reduced to two principle conditions; namely, (i) conditions on interpretation at the Encyclopedia, and (ii) conditions on realization at PF. The choice of $n$ with Norwegian roots can be classified as a hard condition; $n$ [GEN: Masc] and $n$ [GEN: Fem] have a list of possible roots that they license. On the other hand, the choice of $n$ with English roots is a soft condition, subject to Haugen's 'rule' which entails variability; i.e., there are different exponents that compete to be lexicalized on $\mathrm{D}$ in these cases. This also means that the mapping from features to exponents is not straightforward as Finally, for English roots, masculine gender is clearly dominant, suggesting a gradual elimination of gender features on $n$.

This line of reasoning ties in with other recent theoretical approaches that call for all features and the functional sequence they occur in being candidates for restructuring in heritage language grammars (Putnam, 2020; Putnam et al., 2019; Scontras et al., 2018). The AmNo data analyzed in Riksem (2017, 2018) show that features can be restructured without a corresponding change to the functional sequence itself. What is of immediate interest for our treatment of complexity is that based on the heuristic that we develop here, we observe instances of increasing and decreasing complexity. Complexity decreases whenever (i) there are fewer features that are acquired and used, and (ii) when this leads to simpler mapping relations between (gender) features and (gender) exponents. The increase 
in complexity occurs when the relationship between gender and declension classes have become less transparent. This is the case in American Norwegian since the three definite suffixes on nouns (declension classes) are retained even though the free-standing gender markers are restructured and on its way to being eliminated.

Ideally, the models we use are not only effective in delivering an accurate description of linguistic data, but also move us towards a better understanding of the inner working of humans' internalized grammars, and potentially some degree of accurate prediction (Polinsky \& Scontras, 2020). The core elements of our system that play an essential role in modeling complexity are listed in (17):

(17) Criteria for establishing complexity:
a. Number of synsem-features;
b. Number of functional projections; and
c. Mapping from synsem-features to exponents

Let us consider each of these in turn. If all features are retained, the three-gender system is intact. In that case, there are no changes to the number of features. If the functional sequence is retained as well, there are no changes to the hierarchical distribution of the features. When both features and the functional sequence are retained, there will be no changes measured against a given baseline. However, some features may be lost. A threegender grammar can lose one feature and become a two-gender grammar, or lose all features and turn into a non-gendered grammar. Furthermore, the mapping from features to exponents can also be changed, and if the mapping requires more rules to be stated, the mapping can be considered more complex. This can also be argued to include the relationship between gender and declension class, as mismatches are likely to require additional rule specification. 
Importantly, though, there are multiple scenarios available depending on the number of features in the given system that is being considered. In addition to features changing, their hierarchical distribution may also change. This is illustrated clearly in the Scontras et al. (2018) study where they argue that number and gender features are fused into one syntactic position: Num. That is, the position of the gender feature changes its location. It is also conceivable that an entire syntactic projection is lost. If one assumes that gender features have their own projection, an alternative analysis to gender moving from $n$ to Num would be that the gender projection is lost and the functional sequence compressed.

In summary, we argue that the list in (17) is a way of conceptualizing possible outcomes as it identifies the possible sources of grammatical divergence. Note that the typology does not preclude other non-grammatical factors playing a role as it is quite obvious from previous research that they do. Nevertheless, isolating the grammatical factors at play in predicting the structure of heritage grammars is an important objective and our hope is that the proposed typology will inspire other work along similar lines.

\subsection{Challenges for endoskeletal approaches}

The decompositional approach to complexity developed in this paper crucially relies on an exoskeletal approach to grammar. In this subsection we briefly consider the difficulties that a 'traditional', endoskeletal approach to the lexicon immediately encounters in (i) attempting to model the patterns of gender assignment in mixed AmNo DPs, and, central our concern here, (ii) producing a systematic account for measuring complexity. This is important because it strengthens the rationale for adopting an exoskeletal approach to begin with.

Our primary line of criticism is directed at the traditional notion of the 'morpheme' in (18): 


\section{(18) Traditional morpheme:}

$[\alpha, / \mathrm{X} /]$

Traditional morphemes store synsem-information (represented as $\alpha$ ) and any associated (morpho)phonological exponency (represented as $/ \mathrm{X} /$ ) as a unified, non-decompositional unit. This stands in direct contrast to the notion of a distributed lexicon that is instantiated through a dynamic computational process rather than the result of the storage of complex combined units of structure and sound.

Let us consider how an endoskeletal may be able to deal with the data discussed here. First and foremost, nouns will have their gender features inherently specified. Since English nouns display gender by way of the Norwegian indefinite article, this entails that an identical copy of all English nouns would need to be specified for gender in the lexicon. Furthermore, some would have to be multiply specified as a speaker can use the same noun with multiple indefinite articles. Thus, an endoskeletal analysis would require multiple specifications of the same lexical items in several ways. Beyond the general issue of whether or not such multiple specification is empirically and theoretically motivated (see Borer (2005b) and Lohndal (2014) for extensive arguments against such specification), there are also other problems with such an analysis. When acquiring English words and English grammar, speakers have no evidence that English words have gender features as English grammar only has referential pronominal gender. Nevertheless, an endoskeletal analysis would require the speaker to postulate a gender feature for all English words. It may be possible to argue that this analysis could be sustained if the gender of all English words were equivalent to a Norwegian translational equivalent. However, we have seen that this is not the case, which undermines such an analysis. Taken together, an endoskeletal analysis requires a number of stipulations that an exoskeletal analysis does not have to make. 


\section{Conclusion}

Perhaps it is a bit teleological to state that modeling complexity in dynamic, complex systems is a complex task to undertake. There are two paths that one could conceivably choose; one that advocates for the storage of multilayered (or multidimensional) units of language (such as words) or the alternative that envisages these complex units resulting from computational processes. Admittedly, some middle ground, or a spectrum of approaches between these two extremes is possible, however we feel that the latter is superior to the former for a number of reasons. Two of them are particularly relevant here: First, relying on core computational operations to create complex objects significantly reduces the amount of idiosyncratic information to be associated with core principles and desiderata in a system. Second, the mapping strategies of information from one domain of grammar to another enables a conceptually-appealing way to establish a heuristic of decomposing complexity as we laid out in $\$ 3$.

As we have argued here, any change in the feature system of a language is not simply a lexical property of a grammar, but rather, is properly understood as a structural one. Bilingual grammars, and in particular heritage grammars, provide us with a unique testing ground which allows us to illustrate the advantages of a distributed lexicon. Here we took a closer look at the realization of gender in mixed DPs American Norwegian, demonstrating the advantages of decomposing complexity from the perspective of a distributed lexicon and conversely some of the pitfalls associated with its endoskeletal counterpart. Decomposing complexity into features, their functional sequences and the mapping between features and exponents provides a first pass at modeling this evasive notion. Moving forward, the model that we sketched out here needs to be tested on a wider range of other phenomena in typologically unrelated languages, including possible innovations. In summary, this approach provides additional evidence for a distributed architecture of 
the lexicon, whereby syntax and its exponents are fundamental separated, being brought together through computational means. 


\section{References}

Aboh, E. O. (2015). The emergence of hybrid grammars: Language contact and change. Cambridge: Cambridge University Press.

Adger, D. (2003). Core syntax. Oxford: Oxford University Press.

Alexiadou, A. (2017). Building verbs in language mixing varieties. Zeitschrift für Sprachwissenschaft, 36, 165-192.

Alexiadou, A., \& Lohndal, T. (2018). Units of language mixing: A cross-linguistic perspective. Frontiers in Psychology, 9, 1719.

Audring, J. (2014). Gender as a complex feature. Language Sciences, 43, 5-17.

Audring, J. (2017). Calibrating complexity: How complex is a gender system? Language Sciences, 60, 53-68.

Audring, J. (2019). Canonical, complex, complicated? In F. Di Garbo, B. Olsson, \& B. Wälchli (Eds.), Grammatical gender and linguistic complexity. volume I: General issues and specific studies (pp. 15-52). Berlin: Language Science Press.

Benmamoun, E., Montrul, S., \& Polinsky, M. (2013). Heritage languages and their speakers: opportunities and challenges for linguistics. Theoretical Linguistics, 39, 129181.

Borer, H. (2003). Exo-skeletal vs. endo-skeletal explanations: Syntactic projections and the lexicon. In J. Moore \& M. Polinsky (Eds.), The nature of explanation in linguistic theory (pp. 31-67). Stanford, CA: CSLI Publications.

Borer, H. (2005a). In name only - structuring sense - Vol. 1. Oxford: Oxford University Press.

Borer, H. (2005b). The normal course of events - structuring sense - Vol. 2. Oxford: Oxford University Press.

Borer, H. (2013). Taking form - structuring sense - Vol. 3. Oxford: Oxford University 
Press.

Bousquette, J., \& Putnam, M. T. (2020). Redefining language death: Evidence from moribund grammars. Language Learning, 70(S1), 188-225.

Branigan, H. P., \& Pickering, M. J. (2017). An experimental approach to linguistic representation. Behavioral and Brain Sciences, e282, 1-61.

Brennan, J. R., \& Hale, J. T. (2019). Hierarchical structure guides rapid linguistic predictions during naturalistic learning. PLos ONE, 14(1), e0207741.

Bresnan, J., \& Mchombo, S. A. (1995). The lexical integrity principle: Evidence from bantu. Natural Language and Linguistic Theory, 13(2), 181-254.

Bruening, B. (2014). Word formation is syntactic: Adjectival passives in English. Natural Language and Linguistic Theory, 32(3), 363-422.

Bruening, B. (2018). The lexicalist hypothesis: Both wrong and superfluous. Language, 94(1), 1-42.

Chomsky, N. (1955/1975). The logical structure of linguistic theory. New York: MIT Humanities Library and London: Plenum Press.

Chomsky, N. (1957). Syntatic Structures. The Hague: Mouton.

Cook, V. (2016). Premises of multi-competence. In V. Cook \& L. Wei (Eds.), The Cambridge handbook of multi-competence (p. 1-25). Cambridge: Cambridge University Press.

Corbett, G. G. (1991). Gender. Cambridge: Cambridge University Press.

Corbett, G. G. (2012). Features. Cambridge: Cambridge University Press.

Dahl, O. (2004). The growth and maintenance of linguistic complexity. Amsterdam: John Benjamins.

DeGraff, M. (2001). On the origin of creoles: A Cartesian critique of "neo"-Darwinian linguistics. Linguistic Typology, 5, 213-311.

Di Garbo, F., \& Miestamo, M. (2019). The evolving complexity of gender agreement 
systems. In F. Di Garbo, B. Olsson, \& B. Wälchli (Eds.), Grammatical gender and linguistic complexity. Volume II: World-wide comparative studies (pp. 15-60). Berlin: Language Science Press.

Ding, N., Melloni, L., Zhang, H., Tian, X., \& Poeppel, D. (2016). Cortical tracking of hierarchical linguistic structures in connected speech. Nature Neuroscience, 19, $158-164$.

Eide, K. M., \& Hjelde, A. (2015). Verb second and finiteness morphology in norwegian heritage language of the american midwest. In B. R. Page \& M. T. Putnam (Eds.), Moribund Germanic Heritage languages in North America: Theoretical perspectives and empirical findings (pp. 64-101). Leiden: Brill.

Embick, D. (2015). The morpheme: a theoretical introduction. Berlin: Mouton de Gruyter.

Embick, D., \& Marantz, A. (2008). Architecture and blocking. Linguistic Inquiry, 39(1), $1-53$.

Embick, D., \& Noyer, R. (2007). Distributed Morphology and the syntax/morphology interface. In G. Ramchand \& C. Reiss (Eds.), Oxford handbook of linguistic interfaces (pp. 289-324). Oxford: Oxford University Press.

Fine, A. B., Jaeger, T. F., Farmer, T. A., \& Qian, T. (2013). Rapid expectation adaptation during syntactic comprehension. PLOS One, 8(10), e77661.

Flom, G. T. (1903). The gender of English loan-nouns in Norse dialects in America; a contribution to the study of the development of grammatical gender. The Journal of English and Germanic Philology, 5(1), 1-31.

Fuchs, Z., Polinsky, M., \& Scontras, G. (2015). The differential representation of number and gender in spanish. The Linguistic Review, 32, 703-737.

Green, D. W., \& Abutalebi, J. (2013). Language control in bilinguals: The adaptive control hypothesis. Journal of Cognitive Psychology, 25(5), 515-530. 
Grimstad, M. B., Riksem, B. R., Lohndal, T., \& Åfarli, T. A. (2018). Lexicalist vs. exoskeletal approaches to language mixing. The Linguistic Review, 35, 187-218.

Gwilliams, L. (2019). How the brain composes morphemes into meaning. Philosophical Transactions of the Royal Society B, 375(20190311).

Halle, M., \& Marantz, A. (1993). Distributed Morphology and the pieces of inflection. In The view from Building 20: Essays in honor of Sylvain Bromberger (pp. 11-76). Cambridge, MA: MIT Press.

Harstuiker, R., Pickering, M., \& Veltkamp, E. (2004). Is syntax separate or shared between languages? cross-linguistic syntactic priming in Spanish-English bilinguals. Psychological Science, 15(6), 409-414.

Haugen, E. (1953). The Norwegian language in America: A study in bilingual behavior Vol. I-II. Philadelphia: University of Pennsylvania Press.

Hjelde, A. (1996). The gender of English nouns in American Norwegian. In P. S. Ureland \& I. Clarkson (Eds.), Language contact across the atlantic (pp. 297-312). Tübingen: Mac Niemeyer Verlag.

Ježek, E. (2016). The Lexicon: An introduction. Oxford: Oxford University Press.

Johannessen, J. B. (2015). The Corpus of American Norwegian Speech (CANS). In B. Megyesi (Ed.), Proceedings of the 20th Nordic Conference of Computational Linguistics (NODALIDA 2015) (pp. 297-300). NEALT Proceedings Series 23.

Johannessen, J. B., \& Larsson, I. (2015). Complexity matters: on gender agreement in heritage Scandinavian. Frontiers in Psychology, 6, 1842.

Julien, M. (2005). Nominal phrases from a Scandinavian perspective. Amsterdam: John Benjamins.

Kayne, R. (2005). Some notes on comparative syntax, with special reference to english and french. In G. Cinque \& R. Kayne (Eds.), The oxford handbook of comparative syntax (pp. 3-69). Oxford: Oxford University Press. 
Kramer, R. (2015). The morphosyntax of gender. Oxford: Oxford University Press.

Kramer, R. (2016). The location of gender features in the syntax. Language and Linguistics Compass, 10, 661-677.

Kroll, J., \& Gollan, H. (2014). Speech planning in two languages: what bilinguals tell us about language production. In M. Goldrick, V. Ferreira, \& M. Miozzo (Eds.), The Oxford handbook of language production (pp. 165-181). Oxford: Oxford University Press.

Lardiere, D. (1998). Dissociating syntax from morphology in a divergent L2 end-state grammar. Second Language Research, 14(4), 359-375.

Lardiere, D. (2008). Feature-assembly in second language acquisition. In J. Liceras, H. Zobl, \& H. Goodluck (Eds.), The role of formal features in second language acquisition (pp. 107-140). New York: Lawrence Erlbaum.

Lohndal, T. (2014). Phrase structure and argument structure: A case study of the syntaxsemantics interface. Oxford: Oxford University Press.

Lohndal, T., Rothman, J., Kupisch, T., \& Westergaard, M. (2019). Heritage language acquisition: What it reveals and why it is important for formal linguistic theories. Language and Linguistics Compass, 13, 1-19.

Lohndal, T., \& Westergaard, M. (2016). Grammatical gender in American Norwegian heritage language: Stability or attrition? Frontiers in Psychology, 7, 344.

Lohndal, T., \& Westergaard, M. (2021). Grammatical gender: Acquisition, attrition, and change. Journal of Germanic Linguistics, 33, 95-121.

Lohndal, T., Westergaard, M., \& Vangsnes, Ø. A. (2020). Verb second in Norwegian: Variation and acquisition. In R. Woods \& S. Wolfe (Eds.), Rethinking verb second (pp. 770-789). Oxford: Oxford University Press.

López, L. (2020). Bilingual Grammar: Toward an integrated model. Cambridge: Cambridge University Press. 
Marantz, A. (2013). No escape from morphemes in morphological processing. Language and Cognitive Processes, 28(7), 905-916.

McWhorter, J. H. (2001). The world's simplest grammars are creole grammars. Linguistic Typology, 5, 125-166.

Miestamo, M. (2006). On the feasibility of complexity metrics. In K. Kerge \& M.-M. Sepper (Eds.), Finest linguistics. Proceedings of the Annual Finnish and Estonian Conference of Linguistics, tallin, may 6-7, 2004 (pp. 11-26). Tallin: TLÚ.

Miestamo, M. (2008). Grammatical complexity in a cross-linguistic perspective. In M. Miestamo, K. Sinnemäki, \& F. Karlsson (Eds.), Language complexity: Typology, contact, change (pp. 23-41). Amsterdam: John Benjamins.

Montrul, S. (2008). Incomplete acquisition in bilingualism. Amsterdam: John Benjamins.

Montrul, S. (2016). The acquisition of heritage languages. Cambridge: Cambridge University Press.

Müller, S., \& Wechsler, S. (2014). Lexical approaches to argument structure. Theoretical Linguistics, 40, 1-76.

Newmeyer, F. J., \& Preston, L. B. (2014). Measuring linguistic complexity. Oxford: Oxford University Press.

Oseki, Y., \& Marantz, A. (2020). Modeling human morphological competence. Frontiers in Psychology, 11:513740.

Picallo, M. C. (1991). Nominals and nominalization in Catalan. Probus, 3, 279-316.

Polinsky, M. (1997). American Russian: Language loss meets language acquisition. In W. Browne, E. Dornisch, N. Kondrashova, \& D. Zec (Eds.), Formal approaches to slavic linguistics (pp. 370-407). Ann Arbor: Michigan Slavic Publications.

Polinsky, M. (2006). Incomplete acquisition: American Russian. Journal of Slavic Linguistics, 14, 191-262.

Polinsky, M. (2008). Gender under incomplete acquisition: Heritage speakers' knowledge 
of noun categorization. Heritage Language Journal, 6, 40-71.

Polinsky, M. (2011). Reanalysis in adult heritage language: a case for attrition. Studies in Second Language Acquisition, 33, 305-328.

Polinsky, M. (2018). Heritage languages and their speakers. Cambridge: Cambridge University Press.

Polinsky, M., \& Scontras, G. (2020). Understanding heritage languages. Bilingualism: Language and Cognition, 23(1), 3-20.

Prévost, P., \& White, L. (2000). Missing surface inflection or impairment in second language acquisition? Second Language Research, 16(1), 103-133.

Putnam, M. T. (2020). One feature-one head: Features as functional heads in language acquisition and attrition. In P. Guijarro-Feuntes \& C. Suárez-Gómez (Eds.), New trends in language acquisition within the generative perspective (pp. 3-26). Dordrecht: Springer.

Putnam, M. T., Carlson, M., \& Reitter, D. (2018). Integrated, not isolated: Defining typological proximity in an integrated multilingual architecture. Frontiers in Psychology, $8: 2212$.

Putnam, M. T., Perez-Cortes, S., \& Sánchez, L. (2019). Language attrition and the Feature Reassembly Hypothesis. In M. S. Schmid \& B. Köpke (Eds.), The Oxford handbook of language attrition (pp. 18-24). Oxford: Oxford University Press.

Rappaport Hovav, M., \& Levin, B. (1998). Building verb meanings. In M. Butt \& W. Geuder (Eds.), The projection of arguments: Lexical and compositional factors (pp. 97-134). Stanford, CA: CSLI Publications.

Riksem, B. R. (2017). Language mixing and diachronic change: American Norwegian Noun Phrases then and now. Languages, 2(3), 29.

Riksem, B. R. (2018). Language mixing in American Norwegian noun phrases. an exoskeletal analysis of synchronic and diachronic patterns (dissertation). NTNU Nor- 
wegian University of Science and Technology.

Riksem, B. R., Grimstad, M., Lohndal, T., \& Åfarli, T. A. (2019). Language mixing within verbs and nouns in American Norwegian. Journal of Comparative Germanic Linguistics, 22(2), 189-209.

Ritter, E. (1993). Where's gender? Linguistic Inquiry, 24, 795-803.

Rødvand, L. I. (2017). Empirical investigations of grammatical gender in American Heritage Norwegian (MA thesis). University of Oslo.

Sampson, G., Gil, D., \& Trudgill, P. (2009). Language complexity as an evolving variable. Oxford: Oxford University Press.

Scontras, G., Fuchs, Z., \& Polinsky, M. (2015). Heritage language and linguistic theory. Frontiers in Psychology, 6:1545, 1-20.

Scontras, G., Polinsky, M., \& Fuchs, Z. (2018). in support of representational economy: Agreement in heritage Spanish. Glossa, 3, 1-29.

Scontras, G., \& Putnam, M. T. (2020). Lesser-studied heritage languages: An appeal to the dyad. Heritage Language Journal, 17, 152-155.

Shannon, C. (1948). A mathematical theory of communication. The Bell System Technical Journal, 27, 379-423.

Song, Y., Do, Y., Lee, J., Thompson, A., \& Waegemaekers, E. (2019). The reality of hierarchical morphological structure in multimorphemic words. Cognition, 183, $183-276$.

Song, Y., Do, Y., Thompson, A. L., Waegemaekers, E. R., \& Lee, J. (2020). Second language users exhibit shallow morphological processing. Studies in Second Language Acquisition, First View, 1-16.

Sorace, A. (2011). Pinning down the concept of "interface" in bilingualism. Linguistic Approaches to Bilingualism, 1, 1-33.

Spencer, A. (2005). Word-formation and syntax. In P. Štekauer \& R. Lieber (Eds.), 
Handbook of word-formation (pp. 73-97). Dordrecht: Springer.

Starke, M. (2009). Nanosyntax: A short primer to a new approach to language. Nordlyd, $36(1), 1-6$.

Starreveld, P., De Groot, A., Rossmark, B., \& Van Hell, J. (2014). Parallel language activation during word processing in bilinguals: evidence from word production in sentence context. Bilingualism: Language and Cognition, 3(17), 258-276.

Stefanich, S., Cabrelli, J., Hilderman, D., \& Archibald, J. (2019). The morphophonology of intraword codeswitching: Representation and processing. Frontiers in Communication, 4:54.

Stump, G. T. (2001). Inflectional morphology: A theory of paradigm structure. Cambridge: Cambridge University Press.

Sudarshan, A., \& Baum, S. R. (2019). Bilingual lexical access: A dynamic operation modulated by word-status and individual differences in inhibitory control. Bilingualism: Language and Cognition, 3(22), 537-554.

Wechsler, S. (2015). Word meaning and syntax: Approaches to the interface. Oxford: Oxford University Press.

Westergaard, M., \& Lohndal, T. (2019). Verb second word order in norwegian heritage language: Syntax and pragmatics. In D. Lightfoot \& J. Havenhill (Eds.), Variable properties in language: Their nature and acquisition (pp. 23-41). Georgetown: Georgetown University Press.

White, L. (2003). Second language acquisition and universal grammar. Cambridge: Cambridge University Press. 